\title{
THE GENERAL WEB OF ALGEBRAIC SURFACES OF ORDER $n$ AND THE INVOLUTION DEFINED BY IT*
}

BY

TEMPLE R. HOLLCROFT

1. Introduction. Webs of quadric surfaces have been studied quite extensively $\dagger$, but, except for finding the characteristics of its jacobian $\ddagger$ the general web has not been treated for $n>2$.

The surfaces of a web are in $(1,1)$ correspondence with the planes of threespace. In the case of a general web of surfaces of order $n$, this correspondence establishes a space involution of order $n^{3}$. For $n=2$, this involution has been treated by Snyder and Sharpe. $\S$

In the present paper, the properties of both the general web of algebraic surfaces of order $n$ and the space involution associated with such a web are obtained.

2. The web. The equation of a web of algebraic surfaces is

$$
\sum \lambda_{i} f_{i}=0 \quad(i=1,2,3,4),
$$

in which the $\lambda_{i}$ are homogeneous parameters and the $f_{i}$ are homogeneous, algebraic functions of order $n$ in the variables $x_{1}, x_{2}, x_{3}, x_{4}$. The general web treated in this paper is such that the coefficients in each of the four $f_{i}$ defining the web are unrestricted, that is, the $f_{i}$ represent non-singular surfaces and the web has no basis points or curves.

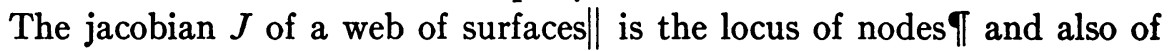
contacts of surfaces of the web. It is a surface of order $4(n-1)$. For a general web, $J$ has no singularities and is, therefore, of genus

$$
D=\frac{1}{3}(2 n-3)(4 n-5)(4 n-7) \text {. }
$$

The characteristics of $J$ result immediately from the Cayley formulas for the characteristics of a non-singular surface.**

\footnotetext{
* Presented to the Society, December 29, 1932; received by the editors February 7, 1933.

$\dagger$ Pascal, Repertorium der höheren Mathematik, vol. $\mathrm{II}_{2}$ (1922), pp. 629-631. Encyklopädie der Mathematischen Wissenschaften, vol. $\mathrm{III}_{2}$, pp. 250-254.

‡ Pascal, loc. cit., p. 680.

\& Virgil Snyder and F. R. Sharpe, Space involutions defined by a web of quadrics, these Transactions, vol. 19 (1918), pp. 275-290.

\| As only algebraic curves and surfaces are treated in this paper, the adjective "algebraic" will be omitted from this point on.

T The term "node" is used to mean an entirely general conic node.

** Pascal, loc. cit., pp. 696-697.
} 
Since a web involves three essential parameters, the surfaces of the web may have contacts or singularities associated with one, two or three invariants. The following singularities on one surface and contacts of surfaces are associated with one, two or three invariants:

I. One invariant. (a) One node or one contact.

II. Two invariants. (a) Two nodes or two contacts. (b) One binode or one stationary contact.

III. Three invariants. (a) Three nodes or three contacts. (b) One binode and one node or one stationary and one simple contact. (c) One special binode $B_{4}$ whose axis has four-point contact with the surface or one contact such that the curve of intersection has a tacnode at the point of contact.

One invariant defines a doubly infinite system of surfaces of the web, the locus of whose singularities or contacts associated with the given invariant is the jacobian surface $J$. Thus $J$ is the locus of nodes and contacts belonging to both doubly infinite systems of surfaces of the web.

Two invariants define a singly infinite system of surfaces of the web, the loci of whose singularities or contacts associated with the two given invariants are curves on $J$. The characteristics of these four curves are obtained in $\$ \S 6$ and 7 .

Three invariants define a finite system of surfaces of the web whose singularities or contacts associated with the three given invariants lie at certain intersections or contacts of the above curves on $J$. The positions and numbers of these for each of the six finite systems are also found in $\$ \S 6$ and 7 .

3. The involution defined by the web.* The $(1,1)$ correspondence existing between the surfaces of the web and the planes of the three-space $(y)$ is defined by the equations

$$
\rho y_{i}=f_{i} \quad(i=1,2,3,4) .
$$

To a line of $(y)$ corresponds a space curve of order $n^{2}$ and genus $(n-1)$ $\left(n^{2}-n-1\right)$, the basis curve of a pencil of surfaces of the web. The surfaces of this pencil are the images of the planes of $(y)$ belonging to a pencil whose axis is the given line.

Conversely, the complete image of a surface of the web or of the basis curve of a pencil of surfaces is the corresponding plane or line of $(y)$ respectively counted $n^{3}$ times.

To a bundle of planes through a point $P$ of $(y)$ corresponds a net of surfaces of the web with $n^{3}$ basis points. These $n^{3}$ basis points are all images of $P$. Conversely, to each of these $n^{3}$ points of $(x)$ corresponds the given point $P$

* The results of this section are chiefly generalizations of those obtained by Snyder and Sharpe (loc. cit.) for $n=2$. 
of $(y)$. The unique correspondence of planes of $(y)$ to surfaces of the web therefore establishes an involution of order $n^{3}$ between the spaces $(x)$ and $(y)$.

The locus of the points of $(y)$, two of whose $n^{3}$ image points coincide, is a surface $L$ called the branch-point surface of the transformation. The corresponding locus of coincidences is $J . L$ and $J$ are in $(1,1)$ correspondence. The order of $L$ is the order of the tact-invariant of two surfaces of the web, which is $4 n^{2}(n-1)$.

The complete image of $L$ is $J$ counted twice and a residual surface $R$ of order $4(n-1)\left(n^{3}-2\right)$. To a point of $L$ correspond two coincident points of $J$ and $n^{3}-2$ distinct points of $R$.

The complete image of $J$ is $L$. The complete image of $R$ is $L$ counted $n^{3}-2$ times.

The image of a plane $\pi$ of $(x)$ is a rational surface $s$ of order $n^{2}$ in $(y)$, whose only singularity is a nodal curve. The image of $s$ is the plane $\pi$ and a residual surface $s_{1}$ of order $n^{3}-1$. The plane $\pi$ meets $J$ in a curve of order $4(n-1)$ through which $s_{1}$ passes. The residual intersection of $\pi$ and $s_{1}$ is a plane curve of order $(n-1)\left(n^{2}+n-3\right)$, the image of the nodal curve of $s$. The nodal curve of $s$ is, therefore, of order $\frac{1}{2} n(n-1)\left(n^{2}+n-3\right)$ and since $s$ is rational, the rank of its nodal curve is $(n-1)\left(n^{2}-3\right)\left(2 n^{3}+2 n^{2}-7 n+2\right) / 3$.

The surface $s$ intersects $L$ in a curve of order $4 n^{4}(n-1)$, consisting of a contact curve of order $4 n(n-1)$, the image of the intersection curve of $\pi$ and $J$; and an intersection curve of order $4 n(n-1)\left(n^{3}-2\right)$, the image of the plane section of $R$ by $\pi$.

The images of linear systems of planes of $(x)$ do not form linear systems of surfaces in $(y)$. The image of a pencil of planes of $(x)$ is a singly infinite, non-linear system of surfaces in $(y)$ of order $n^{2}$. This system of surfaces has in common a curve of order $n$, the image of the line which is the axis of the pencil of planes of $(x)$. This curve $C_{n}$ is rational and has $\frac{1}{2}(n-1)(n-2)$ apparent double points. Any two image surfaces of planes of the pencil intersect in $C_{n}$ and a residual curve $C^{\prime}$ of order $n\left(n^{3}-1\right)$ and genus $n\left(3 n^{3}-5 n^{2}\right.$ $-4 n+7)$ with $\frac{1}{2}(n-1)\left[n(n-1)(n+1)^{2}\left(n^{3}-2\right)-n-2\right]$ apparent double points. $C^{\prime}$ also has $n(n-1)\left(n^{2}-1\right)\left(n^{2}+n-3\right)$ nodes at its intersections with the two nodal curves of the two surfaces.

4. Nets contained in the web. The points of $(y)$, each considered as bearing a bundle of planes, determine a triple infinity of nets of surfaces of the web.

Consider the net $F$ of surfaces corresponding to the bundle of planes through an arbitrary point $P$ of $(y)$. The properties of $F$ are uniquely associated with certain characteristics of the branch-point curve $L_{1}$ of a plane $\pi_{1}$ whose lines are in $(1,1)$ correspondence with the surfaces of $F$. The follow- 
ing characteristics of $L_{1}$ (which will be used in the next section to obtain the characteristics of the branch-point surface $L$ of the web) result on setting $i=3$ in the formulas for a net of hypersurfaces in $i$ dimensions*:

$$
\begin{aligned}
n_{1} & =6 n(n-1)^{2} ; \\
m_{1} & =4(n-1)^{3} ; \\
\delta_{1} & =(n-1)^{2}\left[18 n^{2}(n-1)^{2}-59 n+74\right] ; \\
\kappa_{1} & =12(n-1)^{2}(3 n-4) ; \\
\tau_{1} & =2(n-1)^{2}(n-2)\left(4 n^{3}-8 n^{2}+8 n-25\right) ; \\
\iota_{1} & =30(n-1)^{2}(n-2) .
\end{aligned}
$$

The jacobian curve $J_{1}$ of the net is of order $6(n-1)^{2}$ and is the locus of both nodes and contacts of surfaces of the net. $J$ is also the coincidence curve of the transformation. To nodes $\delta_{1}$, cusps $\kappa_{1}$, bitangents $\tau_{1}$, stationary tangents $\iota_{1}$ of $L_{1}$ correspond uniquely and respectively surfaces of $F$ with two contacts, one stationary contact, two nodes, one binode.

The surfaces of $F$ that have a node are surfaces of the web and therefore all these nodes lie on the surface $J$. The respective jacobian curves of the $\infty^{3}$ nets contained in the web form a triply infinite linear system of curves on $J$, that is, the jacobians of the nets of the web build a web of curves on the jacobian of the web.

Two points $P$ and $P^{\prime}$ of $(y)$ determine the line $P P^{\prime}$ carrying an axial pencil whose planes are common to the two bundles on $P$ and $P^{\prime}$. Corresponding to the two bundles and to their common axial pencil, there are in $(x)$ two sets of surfaces with a pencil of surfaces in common. A pencil of surfaces contains $4(n-1)^{3}$ surfaces with a node. These surfaces belong to both nets and therefore their $4(n-1)^{3}$ nodes lie on both jacobian curves. Therefore, in the web of jacobian curves on $J$, any two jacobian curves intersect in $4(n-1)^{3}$ points.

To a bundle of lines on any point $P$ of $(y)$ corresponds a net of curves of order $n^{2}$ and genus $(n-1)\left(n^{2}-n-1\right)$ with $n^{3}$ basis points. These curves are the intersections of the surfaces of the net corresponding to the bundle of planes on $P$. The nodes of this net of curves lie at contacts of surfaces of the associated net. Any such net of curves has, therefore, the same jacobian curve $J_{1}$ as the associated net of surfaces.

5. The characteristics of $L$. The characteristics of the branch-point surface $L$ will be represented by the following symbols:

$N\left[n^{\prime}\right]$ order [class];

$a\left[a^{\prime}\right]$ order of tangent cone [class of plane section];

\footnotetext{
* Hollcroft, Nets of manifolds in i dimensions, Annali di Matematica, (4), vol. 5 (1927-28), p. 265.
} 
$\kappa^{\prime}\left[\delta^{\prime}\right]$ number of inflections [bitangents] of plane section;

$\kappa[\delta]$ number of cuspidal [nodal] lines of tangent cone;

$b[c]$ order of nodal [cuspidal] curve;

$b^{\prime}\left[c^{\prime}\right]$ class of bitangential [spinodal] developable;

$q\left[q^{\prime}\right]$ class [order] of nodal curve [bitangential developable];

$r\left[r^{\prime}\right]$ class [order] of cuspidal curve [spinodal developable];

$\beta[\gamma](i)$ number of intersections of nodal and cuspidal curves which are cusps on cuspidal [nodal] (neither) curve;

$\beta^{\prime}\left[\gamma^{\prime}\right]\left(i^{\prime}\right)$ number of common planes of bitangential and spinodal developables which are stationary on the spinodal [bitangential] (neither) developable;

$t\left[t^{\prime}\right]$ number of triple points [planes] of nodal curve [bitangential developable];

$\rho^{\prime}\left[\sigma^{\prime}\right]\left(\phi^{\prime}\right)$ order of bitangential [spinodal] (flecnodal) curve;

$\rho[\sigma]$ class of nodal [cuspidal] developable.

The order of $L$ was found in $\S 3$. The class of $L$ is the order of the discriminant of a pencil of surfaces of the web, which is $4(n-1)^{3}$.

The characteristics of the tangent cone to $L$ from any point will now be obtained.

To a bundle of planes of $(y)$ on an arbitrary point $P$ corresponds a net $F$ of surfaces of the web. Of the $\infty^{2}$ planes through $P$, a single infinity are tangent to $L$, enveloping the tangent cone to $L$ from $P$. The planes through $P$ tangent to $L$ correspond uniquely to the single infinity of surfaces of the net that have a node. The planes enveloping the tangent cone to $L$ from $P$ are, therefore, in $(1,1)$ correspondence with the points of the jacobian curve $J_{1}$ of $F$.

The plane section $L_{1}$ of this tangent cone made by any plane $\pi_{1}$ not through $P$ is enveloped by lines which are sections by $\pi_{1}$ of the enveloping tangent planes of the cone. The lines enveloping $L_{1}$ are thus in $(1,1)$ correspondence with the points of $J_{1}$. Therefore, since the lines of $\pi_{1}$ (sections by $\pi_{1}$ of the planes of the bundle) are in $(1,1)$ correspondence with the surfaces of $F$, the plane section $L_{1}$ of the tangent cone is the branch-point curve in the transformation of the lines of $\pi_{1}$ into the surfaces of $F$. From the preceding section, the order of $L_{1}$ is $6 n(n-1)^{2}$ which is, therefore, the order $a$ of the tangent cone to $L$ from any point.

The number of bitangent [stationary] planes from $P$ to $L$ is the class $b^{\prime}$ [ $\left.c^{\prime}\right]$ of the bitangential [spinodal] developable of $L$. These planes are cut by $\pi_{1}$ in bitangents [stationary tangents] to the plane curve $L_{1}$. The number of bitangents $\tau_{1}$ and stationary tangents $\iota_{1}$ of $L_{1}$ were obtained in the preceding section. These values of $\tau_{1}$ and $\iota_{1}$ are, therefore, the values of $b^{\prime}$ and $c^{\prime}$ respectively. 
The number of nodes [cusps] of the plane section $L_{1}$ is the number of nodal [cuspidal] generators $\delta[\kappa]$ of the tangent cone. The number of nodes $\delta_{1}$ and cusps $\kappa_{1}$ of $L_{1}$ were obtained in the preceding section. These are the values of $\delta$ and $\kappa$ respectively.

Since, for any algebraic surface, $a=a^{\prime}$, the class of a plane section of $L$ is $6 n(n-1)^{2}$.

To find the genus of a plane section of $L$, consider the plane section $L^{\prime}$ of $L$ made by any plane $\pi^{\prime} . L^{\prime}$, of order $4 n^{2}(n-1)$, has for its image in $(x)$ the space curve $J^{\prime}$ which is the complete intersection of $J$ and the surface of the web which is the image of $\pi^{\prime}$. This curve $J^{\prime}$ is of order $4 n(n-1)$ and has $2 n(n-1)^{2}(4 n-5)$ apparent double points. Its genus $p^{\prime}$ is, therefore, $p^{\prime}=2 n(n-1)(5 n-8)+1$ which is also the genus of the plane section $L^{\prime}$ of $L$ since $L^{\prime}$ and $J^{\prime}$ are in $(1,1)$ correspondence.

Knowing the order, class and genus of a plane section of $L$, the number of its nodes, cusps, bitangents $\delta^{\prime}$, inflections $\kappa^{\prime}$ can be found by means of Plücker's equations. The numbers of its nodes and cusps are respectively the orders $b$ and $c$ of the nodal and cuspidal curves of $L$.

The genus $D$ of $L$ is the same as that found for $J$ in $\S 3$, since $L$ and $J$ are in $(1,1)$ correspondence. $L$ has no singularities other than a nodal and a cuspidal curve.

In the above, the values of the following characteristics of $L$ have been obtained: $N, n^{\prime}, a, a^{\prime}, b, b^{\prime}, c, c^{\prime}, \delta, \delta^{\prime}, \kappa, \kappa^{\prime}, D$. From these, by use of the Cayley-Zeuthen equations, ${ }^{*}$ the values of $\rho, \rho^{\prime}, \sigma, \sigma^{\prime}, \phi^{\prime}$ result immediately and the remaining characteristics are found by the solution of sets of linear equations.

The characteristics of $L$ obtained above are as follows:

$$
\begin{aligned}
& N=4 n^{2}(n-1) ; \\
& n^{\prime}=4(n-1)^{3} ; \\
& a^{\prime}=a=6 n(n-1)^{2} ; \\
& b=2 n(n-1)\left(4 n^{4}-4 n^{3}-19 n+21\right) ; \\
& b^{\prime}=2(n-1)^{2}(n-2)\left(4 n^{3}-8 n^{2}+8 n-25\right) ; \\
& c=2 n(n-1)(11 n-13) ; \\
& c^{\prime}=30(n-1)^{2}(n-2) ; \\
& \delta=(n-1)^{2}\left[18 n^{2}(n-1)^{2}-59 n+74\right] \\
& \delta^{\prime}=n(n-1)\left[18 n(n-1)^{3}-47 n+69\right] \\
& \kappa=12(n-1)^{2}(3 n-4) ;
\end{aligned}
$$

* Salmon, Geometry of Three Dimensions, 4th edition, 1882, pp. 596-600; Pascal, loc. cit., pp. 692-693. 


$$
\begin{aligned}
\kappa^{\prime} & =4 n(n-1)(7 n-11) \\
\sigma & =2(n-1)^{2}(19 n-22) \\
\sigma^{\prime} & =10 n(n-1)(3 n-5) \\
\rho & =4(n-1)^{2}\left(6 n^{4}-6 n^{3}-31 n+34\right) \\
\rho^{\prime} & =4 n(n-1)\left[6(n-1)^{4}-25 n+39\right] \\
\phi^{\prime} & =2 n(n-1)(73 n-111) \\
r & =4(n-1)\left(26 n^{2}-62 n+37\right) \\
\gamma^{\prime} & =20(n-1)\left(6 n^{2}-18 n+13\right) ; \\
\beta & =8(n-1)(3 n-4)(6 n-7) ; \\
\beta^{\prime} & =40(n-1)(2 n-3)(3 n-7) ; \\
q & =4(n-1)\left[6 n^{3}(n-1)^{2}-49 n^{2}+110 n-62\right] \\
q^{\prime} & =4(n-1)\left[6 n(n-1)^{4}-55 n^{2}+154 n-105\right] \\
\gamma & =8(n-1)\left(11 n^{5}-24 n^{4}+13 n^{3}-87 n^{2}+207 n-123\right) ; \\
\gamma^{\prime} & =120(n-1)\left(n^{5}-6 n^{4}+14 n^{3}-25 n^{2}+42 n-31\right) ; \\
t & =\frac{8}{3}(n-1)\left[4 n^{6}(n-1)^{2}-57 n^{5}+120 n^{4}-63 n^{3}+250 n^{2}-574 n+330\right] \\
t^{\prime} & =\frac{8}{3}(n-1)\left[4(n-1)^{8}-75 n^{5}+438 n^{4}-1002 n^{3}+1498 n^{2}-1937 n+1256\right] \\
i & =i^{\prime}=0 ; \\
D & =\frac{1}{3}(2 n-3)(4 n-5)(4 n-7)
\end{aligned}
$$

6. Loci of contacts and coincidences. If the vertex $P$ of a bundle of planes of $(y)$ is on $L$, at the image point $P_{1}$ on $J$ the surfaces of the corresponding net have a common tangent line $l$ which lies in the tangent plane to $J$ at $P_{1}$. This line $l$ is tangent at $P_{1}$ to all the image curves of all the lines through $P$.

Of this net, the surfaces of a pencil corresponding to the planes of a pencil whose axis is tangent to $L$ at $P$ have contact at $P_{1}$. Since in the tangent plane to $L$ at $P$, all the lines of the flat pencil with vertex at $P$ are tangent to $L$ at $P$, and since the axial pencils on the lines of this flat pencil include all the planes of the bundle on $P$, the net of surfaces which have contact with a line at $P_{1}$ is composed of a simply infinite linear system of pencils of surfaces such that the surfaces of each pencil have contact with each other at $P_{1}$, but do not have contact (except along the line) with any surface of another pencil. Of these surfaces, the one surface common to all the pencils, viz., the surface corresponding to the tangent plane to $L$ at $P$, has a node at $P_{1}$.

The images on $J$ of the cuspidal curve $c$ and the nodal curve $b$ of $L$ are curves of orders $c_{1}$ and $b_{1}$ respectively. 
If $P$ lies on the cuspidal curve $c$ of $L$, the surfaces of the corresponding net all osculate the curve $c_{1}$ at the image point $P_{1}$. Of this net, the surfaces of the pencil corresponding to the planes of the pencil whose axis is the tangent to $c$ at $P$, all have stationary contact with each other at $P_{1}$.

If the surfaces of a net have a common osculating curve at a point $P_{1}$, three of the $n^{3}$ basis points of the net occur at $P_{1}$. Then the cuspidal curve of $L$ is the locus of points $P$ such that at each image point $P_{1}$ on $J$ occurs

(1) the coincidence of three images of $P$;

(2) one stationary contact of the surfaces of a pencil of the web.

If $P$ lies on the nodal curve $b$ of $L$, the surfaces of the corresponding net all have two distinct contacts with the image curve $b_{1}$. One of these contacts is at $P_{1}$ on $J$, the image of $P$ considered on one sheet of $L$ through $b$; the other is at $P_{2}$ on $J$, the image of $P$ considered on the other sheet of $L$ through $b$. Of this net, the surfaces of the pencil corresponding to the planes through the tangent line to $b$ at $P$ all have contact with each other at $P_{1}$ and at $P_{2}$. Of this pencil, one surface corresponding to the tangent plane at $P$ to one sheet of $L$ has a node at $P_{1}$ and another surface corresponding to the tangent plane at $P$ to the other sheet of $L$ has a node at $P_{2}$.

If all the surfaces of a net have two distinct contacts with a curve at $P_{1}$ and $P_{2}$, two of the $n^{3}$ basis points coincide at $P_{1}$ and also two at $P_{2}$. Then the nodal curve of $L$ is the locus of points $P$ such that at each of the two distinct image points $P_{1}$ and $P_{2}$ of $J$ occur

(1) two coincident images of $P$;

(2) one contact of the surfaces of a pencil of the web.

Since the points of $J$ at which occur more than a simple coincidence, or at which occur a combination of simple coincidences, must lie also on the residual surface $R$, they must lie on the curves common to these two surfaces. The images of the nodal and cuspidal curves of $L$ must, therefore, be the curves in which $J$ and $R$ intersect. The surfaces $J$ and $R$ intersect in a composite curve of order $16(n-1)^{2}\left(n^{3}-2\right)$.

The complete image of the nodal curve $b$ of $L$ is the intersection curve $b_{1}$ of $J$ and $R$ of order

$$
b_{1}=4(n-1)\left(4 n^{4}-4 n^{3}-19 n+.21\right)
$$

counted twice and a residual curve $b_{2}$ on $R$ of order

$$
b_{2}=2(n-1)\left(n^{3}-4\right)\left(4 n^{4}-4 n^{3}-19 n+21\right),
$$

which is the nodal curve of $R$. The complete images of $b_{1}$ and $b_{2}$ are $b$ counted twice and $n^{3}-4$ times respectively. The curves $b$ and $b_{1}$ are in $(1,2)$ point correspondence. 
The complete image of the cuspidal curve $c$ of $L$ is the contact curve $c_{1}$ of $J$ and $R$ of order

$$
c_{1}=2(n-1)(11 n-13)
$$

counted three times and a residual curve $c_{2}$ on $R$ of order

$$
c_{2}=2(n-1)\left(n^{3}-3\right)(11 n-13),
$$

which is the cuspidal curve of $R$. The complete images of $c_{1}$ and $c_{2}$ are $c$ counted three times and $n^{3}-3$ times respectively. The curves $c_{1}$ and $c$ are in $(1,1)$ correspondence.

From the above discussion of images of points of $c$, it results that the contact curve $c_{1}$ of $J$ and $R$ is the locus both of the three-point coincidences of the involution and of the stationary contacts of pencils of surfaces of the web. The $n^{3}-3$ residual images of each point of $c$ lie on $c_{2}$.

Similarly, from the above discussion of images of points of $b$, it results that the intersection curve $b_{1}$ of $J$ and $R$ is the locus both of the pairs of simple coincidences of the involution and of the contacts at two distinct points of pencils of surfaces of the web. The $n^{3}-4$ residual images of each point of $b$ lie on $b_{2}$.

At a point $P$ on $L$, one of the $\gamma$ intersections of the curves $b$ and $c$ which is a cusp on $b$, the two sheets of $L$ through $c$ are intersected by another sheet of $L$. The bundle of planes through $P$ corresponds to a net of surfaces all of which (1) osculate the curve $c_{1}$ at $P_{3}$, the image of $P$ considered as on the two sheets of $L$ through $c$ that have a common tangent plane, and (2) have contact with $b_{1}$ at $P_{2}$, the image of $P$ considered as on the other sheet of $L$. Of the $n^{3}$ images of such a point $P$, three coincide at $P_{3}$ and two at $P_{2}$. The planes of the pencil whose axis is the cuspidal tangent to $b$ at $P$ correspond to surfaces of a pencil of the web which have stationary contact at $P_{3}$ and simple contact at $P_{2}$.

Therefore, the number of pencils of surfaces of the web that have two distinct contacts, one of which is stationary, and also the number of pairs of coincidences, each pair consisting of one three-point and one two-point coincidence, is

$$
\gamma=8(n-1)\left(11 n^{5}-24 n^{4}+13 n^{3}-87 n^{2}+207 n-123\right) .
$$

The points $P_{3}$ lie at intersections of the curves $b_{1}$ and $c_{1}$ and the points. $P_{2}$ lie on $b_{1}$. The curve $c_{2}$ passes through the points $P_{3}$ and $b_{2}$ passes through both the points $P_{3}$ and $P_{2}$. Then $c_{2}$ intersects $J$ in $\gamma$ points at the points $P_{3}$, and $b_{2}$ intersects $J$ in $2 \gamma$ points at the points $P_{3}$ and $P_{2}$.

The $n^{3}-5$ residual images of each of the $\gamma$ points of $L$ lie at $n^{3}-5$ intersections $\gamma_{2}$ of $b_{2}$ and $c_{2}$ which are cusps on $b_{2}$. The number $\gamma_{2}=\left(n^{3}-5\right) \gamma$. 
Consider a point $P$ on $L$ at one of the $\beta$ intersections of $b$ and $c$ which are cusps on $c$. At such a point, there is but one sheet of $L$. A plane through $P$ intersects $L$ in a curve with a triple point at $P$ whose penultimate form consists of one node and two cusps. The bundle of planes on $P$ corresponds to a net of surfaces all of which have four-point contact with $c_{1}$ at the image point $P_{4}$ on $J$. The pencil of planes whose axis is the cuspidal tangent to $c$ at $P$ corresponds to the pencil of surfaces which have tacnodal contact at $P_{4}$.

The number of pencils of surfaces of the web that have tacnodal contact and also the number of four-point coincidences of the involution is, therefore,

$$
\beta=8(n-1)(3 n-4)(6 n-7) .
$$

A four-point coincidence may be considered as (1) the union of two simple coincidences or (2) the addition of one image point to a triple coincidence. In case (1), the surfaces of a net which were all tangent to $b_{1}$ at two distinct points would all osculate $b_{1}$ at a single point. In case (2), the surfaces all of which osculated $c_{1}$ at one point would have four-point contact with $c_{1}$ at that point. Since both these possibilities must be accounted for, at the points $P_{\mathbf{4}}$, $b_{1}$ osculates $c_{1}$. Since the three image points in the component triple coincidence of case (2) are symmetrical, the curve $c_{2}$ also osculates $c_{1}$ at the points $P_{4}$, and therefore $c_{2}$ has $3 \beta$ intersections with $J$ at these points. The curve $b_{2}$ does not pass through the points $P_{4}$.

The $n^{3}-4$ residual intersections of each of the $\beta$ points of $L$ lie at $n^{3}-4$ intersections $\beta_{2}$ of $b_{2}$ and $c_{2}$ which are cusps on $c_{2}$. The number $\beta_{2}=\left(n^{3}-4\right) \beta$.

A point $P$ on $L$ at a triple point of $b$ corresponds to three distinct points $P_{2}, P_{2}^{\prime}, P_{2}^{\prime \prime}$ on $b_{1}$. To a bundle of planes through $P$ corresponds a net of surfaces all of which have three distinct contacts with $b_{1}$ at $P_{2}, P_{2}^{\prime}, P_{2}^{\prime \prime}$. Of the $n^{3}$ basis points of the net, two coincide at each of these three points, that is, such a point $P$ has three pairs of coincident image points.

A triple point $P$ of $b$ is also a triple point of $L$. Then at $P$ there exists a single infinity of tangent planes to $L$ enveloping the cubic tangent cone. At the three image points $P_{2}, P_{2}^{\prime}, P_{2}^{\prime \prime}$ of $P$, occur, therefore, nodes belonging to three singly infinite sets of distinct surfaces of the web corresponding to the tangent planes to the three respective sheets of $L$ through $P$.

To the three tangent lines to $b$ at $P$ correspond three pencils of surfaces such that the surfaces of the pencils have two contacts at $P_{2}, P_{2}^{\prime} ; P_{2}, P_{2}^{\prime \prime}$; $P_{2}^{\prime}, P_{2}^{\prime \prime}$ respectively. One plane is common to each of the three pairs of axial pencils. These three planes correspond to three surfaces that have contact at all three image points.

Therefore the number of sets of surfaces, each set containing three surfaces that have three distinct contacts with each other, and also the number 
of sets of three distinct simple coincidences in the involution, is the number of triple points of $b$,

$t=\frac{8}{3}(n-1)\left[4 n^{6}(n-1)^{2}-57 n^{5}+120 n^{4}-63 n^{3}+250 n^{2}-547 n+330\right]$.

The curve $b_{2}$ intersects $b_{1}$ at each of the three coincidences. Then at these points, $b_{2}$ intersects $J$ in $3 t$ points.

The residual images of the $t$ points of $L$ lie at $t_{2}=\left(n^{3}-6\right) t$ triple points of $b_{2}$.

The tangent planes at the points $\beta$ and $\gamma$ of $L$ are non-singular. To a tangent plane at a point $\beta$ corresponds a surface with a node at the image point $P_{4}$. To the two tangent planes at a point $\gamma$ correspond two distinct surfaces, one with a node at $P_{3}$ and the other with a node at $P_{2}$.

The curve $c_{2}$ intersects $J$ in

$$
3 \beta+\gamma=8(n-1)^{2}\left(n^{3}-3\right)(11 n-13)
$$

points, and the curve $b_{2}$ intersects $J$ in

$$
3 t+2 \gamma=8(n-1)^{2}\left(n^{3}-4\right)\left(4 n^{4}-4 n^{3}-19 n+21\right)
$$

points, which occur as described above on the intersection curve $b_{1}$ and the contact curve $c_{1}$ of $J$ and $R$.

7. Loci of singularities of surfaces of the web. As originally defined, the locus of the nodes of the surfaces of the web is $J$.

To a bitangent plane of $L$ corresponds a surface of the web with two nodes. The two points of contact on $L$ correspond respectively and uniquely to the two nodes of the image surface. The locus of the contacts of bitangent planes of $L$ is the bitangential curve $\rho^{\prime}$ of order

$$
\rho^{\prime}=4 n(n-1)\left[6(n-1)^{4}-25 n+39\right] .
$$

The locus of the pairs of nodes, each pair of which belongs to one surface, is a curve $\rho_{1}^{\prime}$ on $J$, the image of $\rho^{\prime}$. The complete image of $\rho^{\prime}$ is the curve $\rho_{1}^{\prime}$ of order

$$
\rho_{1}^{\prime}=4(n-1)\left[6(n-1)^{4}-25 n+39\right]
$$

counted twice and a residual curve $\rho_{2}^{\prime}$ on $R$ of order

$$
\rho_{2}^{\prime}=4(n-1)\left(n^{3}-2\right)\left[6(n-1)^{4}-25 n+39\right] .
$$

The curves $\rho^{\prime}$ and $\rho_{1}^{\prime}$ are in $(1,1)$ correspondence.

The number of intersections of $\rho_{1}^{\prime}$ and $\rho_{2}^{\prime}$ is

$$
16(n-1)^{2}\left(n^{3}-2\right)\left[6(n-1)^{4}-25 n+39\right] \text {. }
$$

To a stationary plane of $L$ corresponds a surface of the web with a binode, the binode being the image point on $J$ of the point of contact on $L$. The locus 
of the contacts of stationary tangent planes to $L$ is the parabolic or spinodal curve $\sigma^{\prime}$ of order

$$
\sigma^{\prime}=10 n(n-1)(3 n-5) .
$$

The complete image of $\sigma^{\prime}$ is the curve $\sigma_{1}^{\prime}$ on $J$ of order

$$
\sigma_{1}^{\prime}=10(n-1)(3 n-5)
$$

counted twice and a residual curve $\sigma_{2}^{\prime}$ on $R$ of order

$$
\sigma_{2}^{\prime}=10(n-1)\left(n^{3}-2\right)(3 n-5) \text {. }
$$

The curve $\sigma_{1}^{\prime}$ on $J$ is the locus of binodes of surfaces of the web.

The number of intersections of $\sigma_{1}^{\prime}$ and $\sigma_{2}^{\prime}$ is

$$
40(n-1)^{2}\left(n^{3}-2\right)(3 n-5) .
$$

The flecnodal curve $\phi^{\prime}$ of order $2 n(n-1)(73 n-111)$ is the locus of contacts of flecnodal tangent planes of $L$. To a flecnodal tangent plane, corresponds a surface of the web with a node, belonging to a pencil of surfaces whose basis curve (the image of the inflectional tangent) osculates the tangent quadric cone of the node at its vertex. To a biflecnodal tangent plane whose contact occurs at a node of $\phi^{\prime}$, corresponds a surface with a node. This surface is common to two pencils of surfaces both of whose basis curves osculate the tangent quadric cone of the node at its vertex. While these singularities account for two and three invariants respectively, actually the only singularity on the image surface in each case is a node. The nodes of the image surfaces of flecnodal and biflecnodal tangent planes of $L$ all lie on a curve $\phi_{1}^{\prime}$ of order $2(n-1)(73 n-111)$, the image on $J$ of $\phi^{\prime}$.

To a triple tangent plane of $L$ corresponds a surface of the web with three nodes. The three contacts on $L$ correspond respectively and uniquely to the three nodes of the image surface. The number of surfaces of the web each of which has three nodes is the number $t^{\prime}$ of triple tangent planes to $L$. This number is

$t^{\prime}=\frac{8}{3}(n-1)\left[4(n-1)^{8}-75 n^{5}+438 n^{4}-1002 n^{3}+1498 n^{2}-1937 n+1256\right]$.

These $3 t^{\prime}$ nodes lie at definite points, possibly nodes, of the curve $\rho_{1}^{\prime}$ on $J$.

To the nodo-cuspidal planes (tangent planes with two contacts one of which is stationary) of $L$ correspond surfaces of the web each with one node and one binode. The node and the binode correspond respectively and uniquely to the simple and stationary contact. The nodo-cuspidal planes of $L$ are the stationary planes $\gamma^{\prime}$ of the bitangential developable. Therefore the number of surfaces of the web that have both a node and a binode are

$$
\gamma^{\prime}=120(n-1)\left(n^{5}-6 n^{4}+14 n^{3}-25 n^{2}+42 n-31\right) .
$$


To the tacnodal tangent planes of $L$ correspond surfaces of the web each of which has a special binode $B_{4}$ whose axis has four-point contact with the surface. The tacnodal contacts and the binodes $B_{4}$ are in $(1,1)$ correspondence. The tacnodal tangent planes of $L$ are the stationary planes $\beta^{\prime}$ of the spinodal developable. Therefore the number of surfaces of the web with a $B_{4}$ is

$$
\beta^{\prime}=40(n-1)(2 n-3)(3 n-7) \text {. }
$$

At the tacnodal points $\beta^{\prime}$ of $L$, the three curves $\rho^{\prime}, \sigma^{\prime}$ and $\phi^{\prime}$ all have the tacnodal tangent as tangent and therefore all three have contact with each other at these points. The cuspidal curve $c$ also passes through each point $\beta^{\prime}$, intersecting each of the above three curves at these points.

Since the cuspidal curve $c$ passes through the $\beta^{\prime}$ tacnodal points of $L$, the $\beta^{\prime}$ binodes $B_{4}$ lie on the contact curve $c_{1}$ of $J$ and $R$ and therefore at the points $B_{4}$, three image points coincide. At these points $\rho_{1}^{\prime}$ and $\sigma_{1}^{\prime}$ are tangent and also the residual curves $\rho_{2}^{\prime}$ and $\sigma_{2}^{\prime}$ have contact with each other and with $\rho_{1}^{\prime}$ and $\sigma_{1}^{\prime}$.

The intersections of $\sigma_{1}^{\prime}$ and $\sigma_{2}^{\prime}$ lie on the curves $c_{1}$ and $b_{1}$ common to $J$ and $R$. The $\beta^{\prime}$ contacts count as $2 \beta^{\prime}$ of these intersections, leaving $40(n-1)$ - $\left[n^{2}(n-1)(3 n-5)-2(n-2)(9 n-13)\right]$ intersections of $\sigma_{1}^{\prime}$ and $\sigma_{2}^{\prime}$ that lie on $b_{1}$. This is likewise the number of intersections of $b$ and $\sigma^{\prime}$ on $L$. To a point $P$ of intersection of $b$ and $\sigma^{\prime}$ correspond one point $P_{1}$ at which occur both a. contact of surfaces of the net along $b_{1}$ and a binode, and another point $P_{2}$ at which occurs only a contact along $b_{1}$. The curves $\sigma_{1}^{\prime}$ and $\sigma_{2}^{\prime}$ intersect only at the points $P_{1}$ of $b_{1}$.

Of the intersections of $\rho_{1}^{\prime}$ and $\rho_{2}^{\prime}, 2 \beta^{\prime}$ lie on $c_{1}$. The remaining $16(n-1)$ $\cdot\left\{(n-1)\left(n^{3}-2\right)\left[6(n-1)^{4}-25 n+39\right]-5(2 n-3)(3 n-7)\right\}$ intersections lie or $b_{1}$ and correspond uniquely to the intersections of $b$ and $\rho^{\prime}$ on $L$. Associated with a point $P$ of intersection of $b$ and $\rho^{\prime}$ is a point $P^{\prime}$ on $\rho^{\prime}$, but not on $b$, which is the other contact of the bitangent plane $\pi$, one of whose contacts is at $P$. The image of $P$ considered on the sheet of $L$ to which $\pi$ is tangent is a point $P_{1}$ of intersection of $\rho_{1}^{\prime}$ and $\rho_{2}^{\prime}$ on $b_{1}$. At $P_{1}$ occurs a node of the image surface $f$ of $\pi$ and also a contact of $f$ and $b_{1}$. At $P_{2}$, the image of $P$ considered on the other sheet of $L$ is simply a contact of $f$ (and the other surfaces of the net) with $b_{1}$. Also, $f$ has a node at $P_{1}^{\prime}$, the image of $P^{\prime}$.

In addition to $\beta^{\prime}$ contacts, the curves $\rho^{\prime}$ and $\sigma^{\prime}$ of $L$ intersect at the $\gamma^{\prime}$ cuspidal points of the nodo-cuspidal planes. The binodes of the $\gamma^{\prime}$ surfaces of the web that have both a node and a binode therefore lie at $\boldsymbol{\gamma}^{\prime}$ intersections of $\rho_{1}^{\prime}$ and $\sigma_{1}^{\prime}$ on $J$, and the associated nodes lie at fixed points on $\rho_{1}^{\prime}$.

8. The steinerian. The jacobian surface $J$ may also be defined as the locus of points $P_{1}$ whose polar planes with respect to all surfaces of the web are concurrent at points $P_{2}$. The points $P_{1}$ are nodes of surfaces of the web. The locus 
of the associated points $P_{2}$ is a surface, the steinerian $S$ of the web. $J$ and $S$ are thus in $(1,1)$ correspondence.

The branch-point surface $L$ is the envelope of planes which are in $(1,1)$ correspondence with the nodes of surfaces of the web which, in turn, are in $(1,1)$ correspondence with the points of $S$. Then the tangent planes of $L$ and the points of $S$ are in $(1,1)$ correspondence. We have, therefore,

The branch-point surface $L$ of the involution defined by the web and the steinerian $S$ of the web are reciprocal surfaces.

All the characteristics of $S$ may be obtained from the characteristics of $L$ given at the end of $\$ 5$ by merely interchanging the accented and unaccented symbols.

9. Webs of quadrics. In a web of quadrics, $J$ and $S$ coincide. $L$ and $J$ are not reciprocal surfaces, however.

Since three conditions are sufficient for a quadric to degenerate into two planes, the web contains a finite number of composite quadrics. This number is ten. The axes of these composite quadrics are ten lines on the quartic $J$. The images of these lines of $J$ are ten conic tropes $C^{\prime}$ of $L . L$ is of order 4 and class 16 . Its reciprocal is a quartic surface with ten nodes.

The simplest method, although one not heretofore used, of obtaining the characteristics of the quadric web and its associated involution, is to determine the characteristics of $L$ by deriving those of its reciprocal ten-nodal quartic. There results the following:

$$
\begin{array}{rlrl}
N & =16, & n^{\prime}=4, \quad a=a^{\prime}=12, & C^{\prime}=10, \\
\delta & =28, \quad \delta^{\prime}=22, \quad \kappa=\kappa^{\prime}=24, & \sigma=32, \quad \rho=80, \\
b & =60, \quad q=40, \gamma=120, & t=80, \\
c & =36, \quad r=68, \beta=80, & \\
\rho^{\prime}=\sigma^{\prime}=b^{\prime}=c^{\prime}=r^{\prime}=\beta^{\prime}=q^{\prime}=\gamma^{\prime}=t^{\prime}=i=i^{\prime}=0 .
\end{array}
$$

Among the general formulas obtained for $L$ in $\$ 5$, all those defining the values of the unaccented characteristics yield the above values for $n=2$; but the correct values (all of which are zero) for $n=2$ can not be obtained from the general formulas for certain accented characteristics, namely, $\rho^{\prime}, \sigma^{\prime}, r^{\prime}$, $\beta^{\prime}, q^{\prime}, \gamma^{\prime}, t^{\prime}$.

The cause of this apparent discrepancy is the existence of the ten conic tropes on $L$ which result from the ten degenerate quadrics of the web. Three conditions are sufficient for an algebraic surface of order $n$ to degenerate only when $n=2$, so that for $n>2$, no general web contains degenerate surfaces. In fact, the most general web of quadrics has the same properties as the polar web of a cubic surface, so that a general web of surfaces exists only for $n \geqq 3$.

Welis College, AURORA, N. Y. 